\title{
AntagoNATs boost gene expression
}

Downregulation of natural antisense transcripts enhances expression of their sense counterparts.

Some diseases are treatable by increasing the levels of certain proteins; neurological disorders, for example, can benefit from an increase in neurotrophins. Unfortunately the administration of recombinant protein, if available, can have drawbacks, from the lack of post-translational modifications to the triggering of an immune response in the recipient. Ideally one would upregulate the endogenous gene, "but," says Claes Wahlestedt from the University of Miami, "there was no easy way to do this." The solution he and his team pursued was to override the cell's own regulatory system to increase the production of a protein of interest.

Wahlestedt has been studying natural antisense transcripts (NATs) for some time. These are RNAs that are transcribed from the opposite strand as a protein coding-gene and overlap it to a certain extent. More and more genes are discovered to have NATs; the current tally stands at some $40 \%$ of all protein-coding genes, though Wahlestedt thinks this number may be an underestimation.

His team focused on brain-derived neurotrophic factor (BDNF), a secreted growth factor important for neuronal growth and maintenance, for which a NAT has been characterized. The researchers reasoned that because the likely role of a NAT is to downregulate the sense transcript, eliminating the NAT could upregulate the gene. They first showed in vitro that this assumption is correct by targeting the NAT with siRNAs. However, to test the effect of NAT knockdown in vivo the team needed a compound that was easier to deliver than siRNAs. They used short, chemically modified, singlestranded oligonucleotides complementary to the NAT, termed antagoNATs, and, upon delivery, they saw a rise in BDNF expression and an increase in neuronal survival and proliferation in the mouse brain.

Investigating the potential mechanism, the researchers found that the BDNF NAT induced repressive chromatin marks that prevented the $B D N F$ gene from being transcribed above a certain level. Removal of the NAT by the antagoNAT prevented the formation of these marks and thus continued transcription of the gene.

With NATs being as common as they are, knocking them down may be a general way to increase expression of many endogenous target genes.

\section{Nicole Rusk}

\section{RESEARCH PAPERS}

Modarresi, F. et al. Inhibition of natural antisense transcripts in vivo results in gene-specific transcriptional upregulation. Nat. Biotechnol. advance online publication (25 March 2012). 